\title{
Hepatic inflammatory cytokine production can be regulated by modulating sphingomyelinase and ceramide synthase 6
}

\author{
MIN HEE KIM ${ }^{1 *}$, HEE KYUNG AHN ${ }^{2 *}$, EUN-JI LEE ${ }^{1}$, SU-JEONG KIM $^{1}$, \\ YE-RYUNG KIM ${ }^{3}$, JOO-WON PARK ${ }^{3}$ and WOO-JAE PARK ${ }^{1}$ \\ ${ }^{1}$ Department of Biochemistry, School of Medicine, Gachon University, Incheon 406-799; ${ }^{2}$ Division of Haematology
and Oncology, Department of Internal Medicine, Gachon University Gil Medical Centre, Incheon 405-760;
${ }^{3}$ Department of Biochemistry, School of Medicine, Ewha Womans University, Seoul 158-710, Republic of Korea
}

Received May 30, 2016; Accepted December 19, 2016

DOI: $10.3892 / \mathrm{ijmm} .2016 .2835$

\begin{abstract}
Chronic inflammation is associated with the pathogenesis of type 2 diabetes and diabetic complications, and palmitate has been nominated as a candidate for the molecular link between these disorders. Recently, a crucial role of ceramide in inflammation and metabolic diseases has been reported. Therefore, in this study, we investigated whether ceramide formation is involved in palmitate-induced hepatic inflammation in vitro and in vivo. Ceramide can be generated either by the de novo pathway or by sphingomyelin degradation, and six different ceramide synthases (CerS) determine the specific acyl chain length of ceramide in mammals. We examined the roles of CerS and sphingomyelinases (SMases) in the secretion of inflammatory cytokines, such as tumour necrosis factor (TNF)- $\alpha$, interleukin (IL)-1 $\beta$, and IL-6 in Hep3B cells. Among the six CerS, CerS6 overexpression uniquely elevated TNF- $\alpha$ secretion via p38 mitogen-activated protein kinase (MAPK) activation. In addition, the treatment of CerS6 overexpressing cells with palmitate synergistically increased cytokine secretion. However, neither palmitate treatment nor CerS6 overexpression altered lipopolysaccharide (LPS)-induced cytokine secretion. Instead, the activation of acidic (A)-SMase
\end{abstract}

Correspondence to: Dr Woo-Jae Park, Department of Biochemistry, School of Medicine, Gachon University, 155 Gaet-berl-ro, Incheon 21999, Republic of Korea

E-mail: ooze@gachon.ac.kr

Professor Joo-Won Park, Department of Biochemistry, School of Medicine, Ewha Womans University, 911-1 Mok-Dong, Seoul 158-710, Republic of Korea

E-mail: joowon.park@ewha.ac.kr

*Contributed equally

Abbreviations: CerS, ceramide synthase; ERK, extracellular signalregulated kinase; IL, interleukin; JNK, c-Jun N-terminal kinase; SMase, sphingomyelinase; TNF- $\alpha$, tumour necrosis factor- $\alpha$

Key words: ceramide synthase 6, inflammation, acid sphingomyelinase, MAP kinase, tumour necrosis factor- $\alpha$, interleukin was involved in LPS-induced cytokine secretion via the MAPK/ $\mathrm{NF}-\kappa \mathrm{B}$ pathway. Finally, the suppression of ceramide generation via A-SMase inhibition or de novo ceramide synthesis decreased high-fat diet-induced hepatic cytokine production in vivo. On the whole, our results revealed that CerS6 played a role in TNF- $\alpha$ secretion, and palmitate augmented inflammatory responses in pathophysiological conditions in which CerS6 is overexpressed. In addition, A-SMase activation was shown to be involved in LPS-induced inflammatory processes, suggesting that the modulation of CerS6 and A-SMase may be a therapeutic target for controlling hepatic inflammation.

\section{Introduction}

Inflammation is not only a physiological response against deleterious stimuli, such as infection, but also a contributing factor to the pathogenesis of various metabolic disorders. For example, systemic inflammatory markers are associated with the disease progression of type 2 diabetes and its macrovascular complications (1). The inflammation that accompanies metabolic syndrome usually displays a unique characteristic. As it is not accompanied by infection or massive tissue injury, it is often termed 'low-grade' chronic inflammation (2). Scientists have endeavoured to elucidate a mechanistic link between inflammation and metabolic disorders.

The levels of free fatty acids are increased in obesity, and saturated fatty acids have been nominated as candidates that increase the inflammatory response in metabolic syndrome (3). In particular, palmitic acid levels in plasma and triglycerides correlate with insulin resistance (4), and a recent study revealed that palmitate activates inflammatory pathways, leading to an impairment in insulin transcytosis (5). Along with palmitate, significant increases in plasma ceramide levels have also been observed in patients with type 2 diabetes and in diabetic animals, and these increases further correlate with the progression of diabetes and the activation of inflammatory mediators, such as tumour necrosis factor (TNF)- $\alpha(6,7)$.

Ceramide can be generated either by attaching fatty acyl CoA to a long-chain base or by degrading pre-existing sphingolipids, such as sphingomyelin (8). Six mammalian ceramide synthases (CerS) determine the acyl chain length of ceramide $(8,9)$. CerS1 and CerS5-6 generate ceramides 
with long acyl chains (C18- and C16-ceramides, respectively) (10-12). CerS4 generates C18-C20-ceramides (13), and CerS2 produces ceramides with very long acyl chains (C22-C24-ceramides) (14). CerS3 generates ceramides with even longer acyl chains (C26-C34-ceramides) (15). The distribution of CerS and ceramides with distinct fatty acyl chain lengths has been reported to play different roles in various pathophysiologies $(8,9)$. The overexpression of CerS2 results in partial protection from radiation-induced apoptosis, whereas the overexpression of CerS5 increases the apoptosis of HeLa cells (16). In addition to de novo synthesis, ceramide can also be formed by sphingomyelinase (SMase) activation. SMase degrades sphingomyelin into ceramide and phosphorylcholine and can be either acidic (A-SMase) or neutral (N-SMase), depending on the optimal $\mathrm{pH}$ needed for activation (17).

The liver is a central organ in metabolism that controls lipogenesis, gluconeogenesis and cholesterol. Liver cirrhosis contributes to the fourth leading cause of mortality due to diabetes (18). Considering the important role of the liver in metabolic processes, understanding inflammatory cytokine secretion in the liver would be crucial for the elucidation of the underlying mechanisms of inflammation in metabolic diseases. Therefore, in this study, we examined the role of ceramide formation in hepatic inflammatory cytokine production upon palmitate or lipopolysaccharide (LPS) stimulation in vitro and upon high-fat diet (HFD) feeding in vivo.

\section{Materials and methods}

Materials. The following materials were purchased: fumonisinB1(FB1),LPS, palmitate, myriocin,GW4869,SB203580, SP600125, anti-CerS2 antibody (HPA027262), anti-CerS4 antibody (SAB4301210), anti- $\alpha$-tubulin antibody (T9026) and anti-HA antibody (H6908) (all from Sigma-Aldrich, St. Louis, MO, USA); PD98059 and anti-p38 (8690), anti-phosphorylated (phosphor)-p38 (Thr180/Tyr182) (4511), anti-c-Jun N-terminal kinase (JNK) (9252), anti-phosphorylated (p-)JNK (Thr183/ Tyr185) (9255), anti-extracellular signal-regulated kinase (ERK) (4695), anti-p-ERK (Thr202/Tyr204) (4370), anti-p65 (8242), anti-p-IкB (9246), and anti-Lamin A/C (4777) antibodies (all from Cell Signalling Technology, Inc., Beverly, MA, USA); pyrrolidinedithiocarbamate ammonium (PDTC) (BioVision, Inc., Mountain View, CA, USA); anti-CerS1 (H00010715-A01), anti-CerS5 (PAB13439) and anti-CerS6 (H00253782-A01) antibodies (Abnova, Taipei, Taiwan); anti-mouse-HRP (horseradish peroxidase)(115-036-003) and anti-rabbit-HRP (111-035-003) antibodies (Jackson Laboratory, Ben Harbor, ME, USA).

Cell culture and transfection. Hep3B cells were purchased from the American Type Culture Collection (ATCC, Rockville, MD, USA; HB8064) and were grown in Dulbecco's modified Eagle's medium (HyClone, Logan, UT, USA), which was supplemented with $10 \%$ fetal bovine serum and $1 \%$ penicillin-streptomycin (HyClone).The Hep3B cells were transfected with plasmidsusing Metafectene (Biontex Laboratories $\mathrm{GmbH}$, Munich, Germany), according to the manufacturer's instructions. In some cases, the Hep3B cells were transfected with pCMV-empty vector, 2 or $5 \mu \mathrm{g}$ of pCMV-CerS6-HA plasmid in 6-well plates. All plasmids, including pCMV-empty vector, pCMV-CerS1-HA, CerS2-HA, CerS4-HA, CerS5-HA and CerS6-HA were kindly provided by professor Anthony H. Futerman (Weizmann Institute of Science, Rehovot, Israel).

Animals and HFD feeding. Male C57BL/6J mice (6 weeks old) were purchased from Orient Bio Inc. (Seoul, Republic of Korea), and housed under specific pathogen-free conditions. All animal experiments and procedures were approved by The Animal Ethics Committee at Ewha Womans University College of Medicine (Seoul, Korea; ESM no. 14-0280), and all animals were treated in accordance with the Animal Care Guidelines of Ewha Womans University. The mice were housed in a controlled environment at $21-23^{\circ} \mathrm{C}$ and $51-54 \%$ humidity, with a 12-h light-dark cycle, and were supplied with food and water ad libitum. The mice were fed a HFD (D12492; Research Diets Inc., New Brunswick, NJ, USA) for 6-24 weeks and hepatic CerS levels were analyzed. Other mice were fed a HFD for 24 weeks and administered an intraperitoneal injection of desipramine $(10 \mathrm{mg} / \mathrm{kg} / \mathrm{day})$ and myriocin $(0.15 \mathrm{mg} / \mathrm{kg} / \mathrm{day})$, as previously described $(19,20)$. Three mice were used in each group. The mice were sacrificed by $\mathrm{CO}_{2}$ inhalation at 6,12 , 18 and 24 weeks after being fed the HFD. The mice not fed the HFD were used as controls. The liver tissue was perfused with phosphate-buffered saline through the inferior vena cava, and the portal vein was cut using scissors to remove blood from the liver. The livers were then collected, and snap-frozen in liquid nitrogen for storage at $-80^{\circ} \mathrm{C}$.

Enzyme-linked immunosorbent assay (ELISA). At $24 \mathrm{~h}$ following CerS6-HA transfection or $5 \mu \mathrm{g} / \mathrm{ml}$ LPS treatment and a further $24 \mathrm{~h}$ of co-incubation with various chemicals ( $2 \mu \mathrm{M}$ desipramine, $10 \mu \mathrm{M}$ Fumonisin B1, $10 \mu \mathrm{M}$ GW4869, $10 \mu \mathrm{M}$ SB203580, $10 \mu \mathrm{M}$ SP600125, $10 \mu \mathrm{M}$ PD98059, $10 \mu \mathrm{M}$ PDTC, or $500 \mu \mathrm{M}$ palmitate) the levels of TNF- $\alpha$, interleukin (IL)- $1 \beta$ and IL- 6 in the Hep3B cell culture medium were measured using respective ELISA kits [TNF- $\alpha$ and IL-1 $\beta$ Human ELISA kits (Abfrontier, Seoul, Korea); and the IL-6 Human ELISA kit (Abcam, Cambridge, MA, USA)], according to the manufacturer's instructions. The TNF- $\alpha$ and IL-1 $\beta$ levels in the livers of the HFD-fed mice that were co-treated with desipramine $(10 \mathrm{mg} / \mathrm{kg} /$ day $)$ or myriocin $(0.15 \mathrm{mg} / \mathrm{kg} /$ day $)$ were analysed using a Mouse TNF- $\alpha$ ELISA kit (Biolegend Inc., San Diego, CA, USA) and a Mouse IL-1 $\beta$ ELISA kit (Abcam).

Western blot analysis. The Hep3B cells were lysed using RIPA buffer $(50 \mathrm{mM}$ of Tris- $\mathrm{Cl}, \mathrm{pH} 7.5,150 \mathrm{mM}$ of $\mathrm{NaCl}, 1 \%$ nonidet $\mathrm{P}-40,0.5 \%$ sodium deoxycholate and $0.1 \%$ SDS) containing protease and phosphatase inhibitors (Sigma-Aldrich). The protein levels were then quantified using Protein Assay Dye Reagent (Bio-Rad Laboratories, Hercules, CA, USA). Fifty micrograms of proteins were separated by SDS-PAGE on 10\% SDS-polyacrylamide gels and transferred onto nitrocellulose membranes (Bio-Rad). The membranes were then blocked with $5 \%$ bovine serum albumin (Sigma-Aldrich) in PBST (PBS with 0.1\% Tween-20) for $1 \mathrm{~h}$ and incubated with primary antibodies overnight at $4^{\circ} \mathrm{C}$. Secondary antibodies were attached for $1 \mathrm{~h}$ at room temperature. Protein bands were detected by the Chemidoc MP imaging system (Bio-Rad), using ECL Western Blotting Detection Reagents (Amersham Biosciences, Buckinghamshire, UK). 
Table I. Primers used for qPCR.

\begin{tabular}{ll}
\hline Gene & \multicolumn{1}{c}{ Primer sequences } \\
\hline TNF- $\alpha$ (mouse) & F: 5'-CTGTAGCCCTCGTAGC-3' \\
& R: 5'-TTGAGATCCATGCCGTTG-3' \\
IL-1 $\beta$ (mouse) & F: 5'-TGTAATGAAAGACGGCACACC-3' \\
R: 5'-TCTTCTTTGGGTATTGCTTGG-3' & F: 5'-TCCAGTTGCCTTCTTGGGAC-3' \\
IL-6 (mouse) & R: 5'-GTACTCCAGAAGACCAGAGG-3' \\
& F: 5'-CGACTTCAACAGCAACTCCCACTCTTCC-3' \\
GAPDH (mouse) & R: 5'-TGGGTGGTCCAGGGTTTCTTACTCCTT-3'
\end{tabular}

TNF- $\alpha$, tumour necrosis factor- $\alpha$; IL, interleukin; GAPDH, glyceraldehyde-3-phosphate dehydrogenase.

A-SMase activity assay. A-SMase activity was examined as previously described $(21,22)$. Briefly, the Hep3B cells were lysed in $500 \mu \mathrm{l}$ sodium acetate buffer $(50 \mathrm{mM}$ sodium acetate, $\mathrm{pH} 4.5)$. To initiate the reaction, C6-NBD-SM (1 nmol; Avanti Polar Lipid, Alabaster, AL, USA) was added to the Hep3B cell lysates followed by incubation at $37^{\circ} \mathrm{C}$ for $20 \mathrm{~min}$. The reactions were terminated by the addition of 3 volumes of chloroform:methanol (2:1). NBD-ceramide was separated by thin-layer chromatography using chloroform:methanol:9.8 mM aqueous $\mathrm{CaCl}_{2}(60: 35: 8 ; \mathrm{v} / \mathrm{v} / \mathrm{v})$.

Separation of nuclear and cytoplasmic fractions. The isolation of nuclear and cytoplasmic fractions was performed as previously described (23). Briefly, the Hep3B cells were lysed in fractionation buffer (50 mM HEPES, pH 7.4, $10 \mathrm{mM}$ $\mathrm{KCl}, 1 \mathrm{mM}$ EDTA, $1 \mathrm{mM}$ EGTA, $1 \mathrm{mM}$ dithiothreitol and $0.1 \%$ NP-40) containing protease and phosphatase inhibitors (Sigma-Aldrich). Following $15 \mathrm{~min}$ of incubation on ice, the lysates were centrifuged at $4,000 \mathrm{x} \mathrm{g}$, for $5 \mathrm{~min}$ at $4^{\circ} \mathrm{C}$; and the pellet was further resuspended in lysis buffer $(20 \mathrm{mM}$ HEPES, $\mathrm{pH} 7.4,150 \mathrm{mM} \mathrm{NaCl}, 12.5 \mathrm{mM}$ glycerophosphate, $1.5 \mathrm{mM}$ $\mathrm{MgCl}_{2}, 2 \mathrm{mM}$ EGTA, $10 \mathrm{mM} \mathrm{NaF}, 2 \mathrm{mM}$ dithiothreitol, $1 \mathrm{mM} \mathrm{Na} \mathrm{VO}_{4}, 0.5 \%$ Triton $\mathrm{X}-100$, protease and phosphatase inhibitors) and sonicated to obtain the nuclear fraction. The supernatant was centrifuged at $12,500 \mathrm{x} \mathrm{g}$ for $5 \mathrm{~min}$ at $4^{\circ} \mathrm{C}$ to obtain the cytoplasmic fraction.

Quantitative polymerase chain reaction ( $q P C R$ ). Total RNA from the mouse livers was extracted using RNeasy mini kits (Qiagen,Inc., Valencia, CA, USA), and cDNA from the extracted RNA was synthesized using a Verso cDNA Synthesis kit (Fisher Scientific, Hampton, NH, USA). qPCR was performed using the SYBR-Green Real-Time PCR Master Mix (Life Technologies, Grand Island, NY, USA) in an ABI PRISM 7500 Sequence Detection System (Applied Biosystems Inc., Waltham, MA, USA), as previously described (24). Relative gene expression was calculated by using the $2^{-\Delta \Delta \mathrm{Ct}}$ method (24). The primers used are listed in Table I.

Liquid chromatography-electrospray ionization-tandem mass spectrometry (LC-ESI-MS-MS) analysis of ceramide. Ceramide analyses by LC-ESI-MS-MS were conducted as previously described $(25,26)$ with some modifications. Briefly, lipids extracted with $1 \times 10^{7}$ cells were injected into a HPLC (Agilent 1,200 series; Agilent Technologies, Inc., Santa Clara, CA,USA) and separated through a reverse phase KINETEX C18 column $(2.1 \times 50 \mathrm{~mm}$, ID: $2.6 \mu \mathrm{m})$ (Phenomenex Inc., St. Louis, MO, USA). The HPLC column effluent was then introduced into an API 3200 Triple quadruple mass (AB Sciex, Toronto, $\mathrm{ON}, \mathrm{Canada}$ ) and analysed using electrospray ionization in positive mode with multiple reaction monitoring to select both parent and characteristic daughter ions specific to each analyte simultaneously from a single injection. Data were acquired using Analyst 1.4.2 software (Applied Biosystems).

Statistical analysis. All experiments were repeated at least 3 times independently, and values are presented as the means \pm standard error of the mean. Statistical significance was calculated using the Student's t-test. A value of $\mathrm{P}<0.05$ was considered to indicate a statistically significant difference.

\section{Results}

Palmitate and CerS6have synergistic effects on TNF- $\alpha$ secretion via the phosphorylation of 38 MAPK. To examine the role of de novo ceramide formation in inflammatory cytokine secretion from liver cells, CerS1, CerS2, CerS4, CerS5, or CerS6 was overexpressed in Hep3B cells and the overexpression of each CerS increased the levels of ceramides with different acyl chain lengths successfully (Fig. 1A). CerS3 was excluded, as it was previously shown to be hardly expressed in the liver (14). Only CerS6 overexpression increased TNF- $\alpha$ secretion (Fig. 1B). The overexpression of either CerS had no effect on IL-1 $\beta$ and IL-6 secretion (Fig. 1C and D). As MAPK activation has been reported to be involved in TNF- $\alpha$ secretion (27), we then examined the MAPK pathway following CerS6 overexpression. CerS6 overexpression elevated p38 phosphorylation in a dose-dependent manner, although neither JNK nor ERK was activated upon CerS6 overexpression (Fig. 2A, upper panel). TNF- $\alpha$ secretion increased as the CerS6 expression levels increased (Fig. 2A, lower panel). Of note, a synergistic effect was observed in the cells overexpressing CerS6 treated with palmitate as regards both $\mathrm{C} 16$-ceramide generation and p38 phosphorylation (Fig. 2B). To examine whether p38 is a 

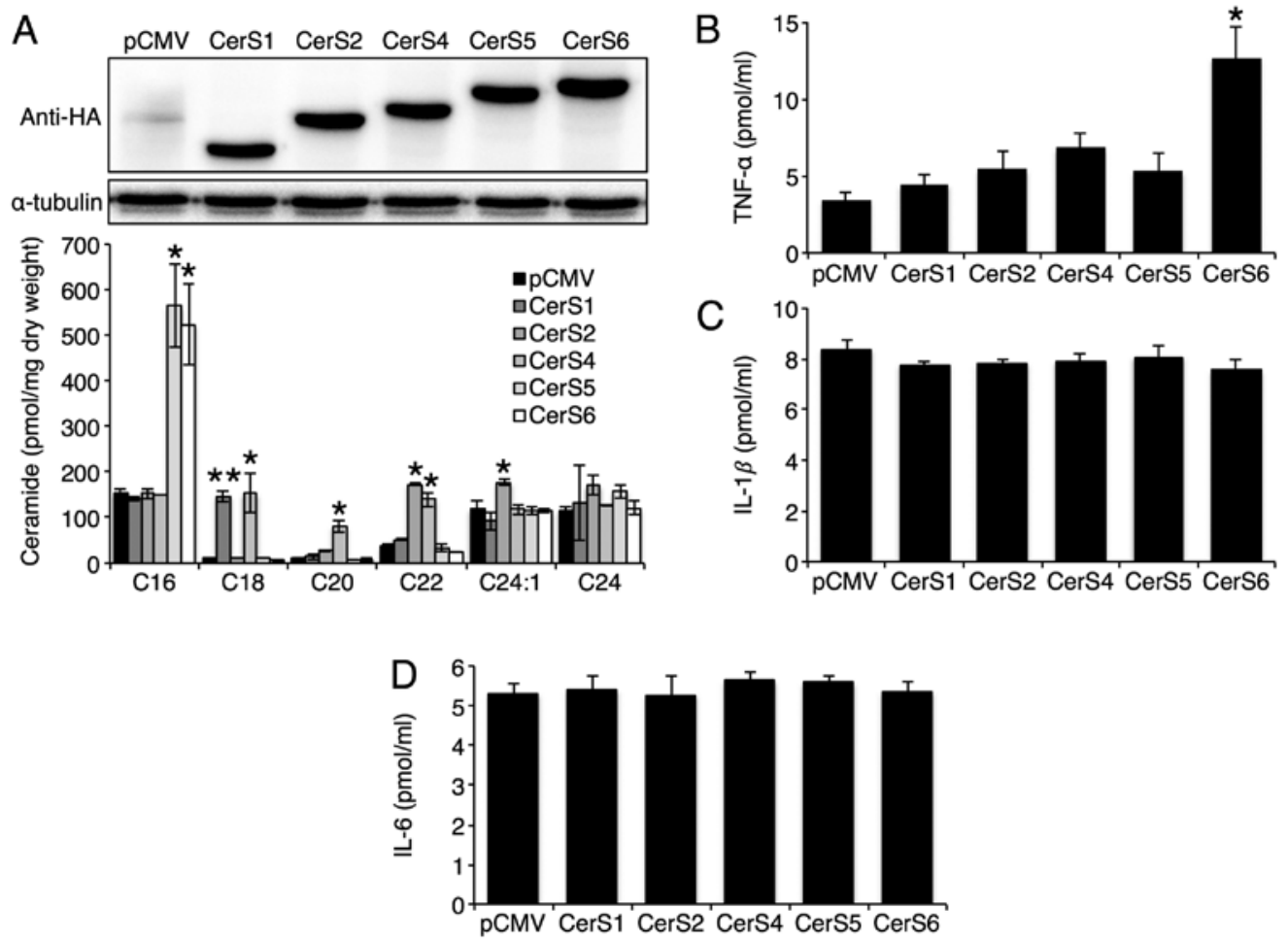

Figure 1. Ceramide synthase 6 (CerS6) overexpression increases tumour necrosis factor (TNF)- $\alpha$ secretion. (A) Representative western blots of CerS1, CerS2, CerS4, CerS5, or CerS6 overexpression (upper panel). Acyl chain lengths of ceramides were measured by LC-ESI-MS-MS following CerS overexpression (lower panel) (n=3). (B) TNF- $\alpha$, (C) interleukin (IL)-1 $\beta$, and (D) IL-6 levels in the Hep3B cell culture medium were measured using enzyme-linked immunosorbent assay (ELISA) kits at $48 \mathrm{~h}$ following CerS overexpression $(\mathrm{n}=3)$. The values are expressed as the means \pm standard error of the mean. ${ }^{*} \mathrm{P}<0.05,{ }^{* *} \mathrm{P}<0.01 \mathrm{compared}$ to transfection with pCMV. Three independent experiments were performed.

direct target of CerS6 upon palmitate treatment, the CerS6 overexpressing cells were co-treated with palmitate and the CerS inhibitor, FB1. CerS inhibition reversed p38 phosphorylation; this effect was similar to that achieved with the p38 inhibitor, SB203580, thus confirming that p38 is downstream of CerS6 (Fig. 2B). In accordance with p38 activation, we also observed a synergistic effect between palmitate treatment and CerS6 overexpression as regards TNF- $\alpha$ secretion (Fig. 2C); treatment with SB203580 and FB1 also reversed the palmitateand CerS6 overexpression-induced increase in TNF- $\alpha$ secretion (Fig. 2C). As the p38/NF- $\mathrm{kB}$ pathway is important for TNF- $\alpha$ secretion (27), we further examined the NF- $\kappa B$ pathway upon palmitate treatment in CerS6 overexpressing Hep3B cells. As expected, palmitate treatment increased nuclear $\mathrm{p} 65$ translocation and IкB phosphorylation; both events were reversed by p38 inhibition (Fig. 2D). Similarly, TNF- $\alpha$ secretion was also inhibited by treatment with PDTC (a NF- $\kappa \mathrm{B}$ inhibitor) (Fig. 2C). Thus, we concluded that palmitate, which is a saturated fatty acid that is increased in metabolic diseases, synergistically increases TNF- $\alpha$ production with CerS6 in liver cells via the CerS6/p38/NF- $\mathrm{KB}$ pathway.

LPS increases TNF- $\alpha$ secretion via sphingomyelin degradation, but not via CerS activation. LPS is the endotoxin that triggers strong inflammatory responses in bacterial infection (28). To examine whether there is a synergistic effect between CerS6 overexpression or palmitate treatment and LPS in cytokine secretion, pCMV (empty vector)-transfected or CerS6 overexpressing cells were co-treated with palmitate and
LPS. Although palmitate treatment alone did not affect TNF- $\alpha$, IL-1 $\beta$ and IL-6 secretion, palmitate synergistically elevated TNF- $\alpha$, IL-1 $\beta$ and IL- 6 secretion in the CerS6 overexpressing Hep3B cells (Fig. 3). As expected, LPS treatment significantly increased TNF- $\alpha$, IL- $1 \beta$ and IL-6 secretion. However, no synergistic effect was observed between LPS and palmitate treatment or CerS6 overexpression as regards cytokine secretion (Fig. 3).

As CerS6 did not affect LPS-induced cytokine secretion, we examined whether ceramide generation via sphingomyelin degradation is involved in LPS-induced cytokine secretion. Treatment with LPS, but not palmitate, increased A-SMase activity (Fig. 4A), and co-incubation with the A-SMase inhibitor, desipramine, reduced total ceramide and LPS-induced TNF- $\alpha$, IL-1 $\beta$ and IL-6 secretion (Fig. 4B-E). This result demonstrated that A-SMase activity played a critical role in LPS-induced cytokine secretion. Although N-SMase activity was not altered upon LPS treatment (data not shown), co-incubation with the N-SMase inhibitor, GW4869, also partially reduced total ceramide and LPS-induced TNF- $\alpha$, IL- $1 \beta$ and IL- 6 secretion (Fig. 4B-E), suggesting that ceramide generation via A-SMase and N-SMase is involved in LPS-induced hepatic cytokine production. Co-incubation with FB1 did not influence LPS-induced cytokine secretion (Fig. 4C-E). Therefore, CerS did not play a critical role in LPS-induced hepatic cytokine secretion.

Activation of the p38 MAPK/NF- $\kappa B$ pathway plays a critical role in hepatic inflammatory cytokine secretion. MAPK 
A

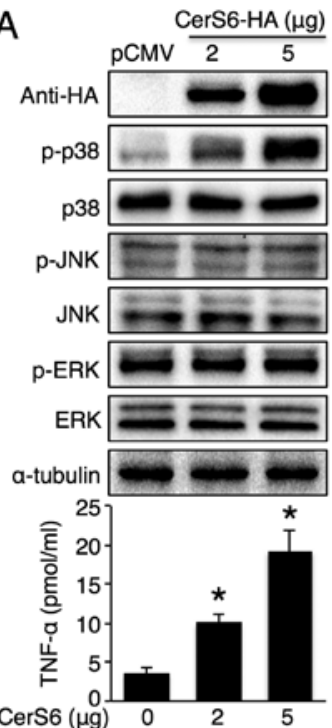

$\mathrm{B}$

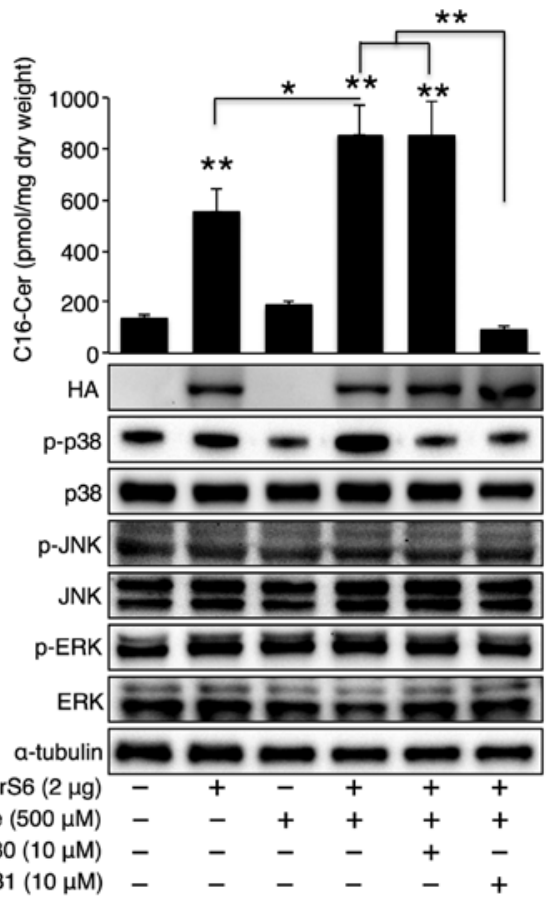

D

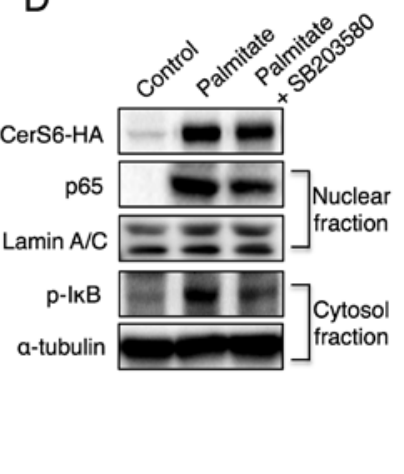

Figure 2. Palmitate treatment exerts a synergistic effect with ceramide synthase 6 (CerS6) on the release of tumour necrosis factor (TNF)- $\alpha$ via the p38/NF- $\mathrm{kB}$ pathway. (A) Representative western blots of mitogen-activated protein kinase (MAPK) pathway-related proteins upon CerS6 overexpression (upper panel). Secreted TNF- $\alpha$ levels were measured in the Hep3B cell culture medium using ELISA kits (lower panel, $\mathrm{n}=3$ ). (B) C16-ceramide levels (upper panel) and representative western blots of MAPK (lower panel) in CerS6 overexpressing Hep3B cells following co-treatment with palmitate, SB203580 (p38 inhibitor), and fumonisin B1 (CerS inhibitor). (C) TNF- $\alpha$ release was measured in the culture medium from CerS6 overexpressing Hep3B cells following co-incubation with palmitate, SB203580, fumonisin B1 and pyrrolidinedithiocarbamate ammonium (PDTC) (NF- $\kappa$ B inhibitor) (n=3). (D) Representative western blots of the NF- $\kappa$ B pathway in CerS6 overexpressing Hep3B cells following co-treatment with palmitate and SB203580. The values are expressed as the means \pm standard error of the mean. ${ }^{*} \mathrm{P}<0.05,{ }^{* *} \mathrm{P}<0.01,{ }^{* * * *} \mathrm{P}<0.001$. Three independent experiments were performed.

activation plays an important role in inflammatory cytokine production (29). Therefore, we examined whether the inhibition of sphingomyelin degradation alters LPS-induced MAPK activation. As expected, LPS elevated the phosphorylation levels of all 3 MAPKs (p38, JNK, and ERK). Co-incubation with desipramine or GW4869 reversed these phosphorylation levels (Fig. 5A), thus suggesting that the MAPK pathway is downstream of sphingomyelin degradation. To further investigate the direct role of MAPK in hepatic cytokine secretion, we co-treated the cells with LPS and SB203580 (a p38 inhibitor), SP600125 (a JNK inhibitor), or PD98059 (an ERK inhibitor). The inhibition of p38 or JNK significantly attenuated the effects of LPS on TNF- $\alpha$, IL- $1 \beta$ and IL- 6 secretion (Fig. 5B-D). However, the inhibition of ERK only reduced IL-1 $\beta$ secretion. Similarly, the inhibition of p38 or JNK, but not ERK, markedly reduced the LPS-induced activation of the NF- $\mathrm{KB}$ signalling pathway. However, the extent of NF- $\mathrm{KB}$ inhibition was greater with p38 inhibition than with JNK inhibition (Fig. 6A). Finally, we directly examined the role of NF- $\mathrm{BB}$ in LPS-induced cytokine production. Co-incubation with LPS and an NF- $\kappa \mathrm{B}$ inhibitor, PDTC, significantly reduced LPS-induced cytokine secretion (Fig. 6B-D). Of note, the direct NF- $\mathrm{NB}$ inhibition further diminished the partial reduction in TNF- $\alpha$ and IL-6 levels by JNK inhibition without affecting cytokine levels by p38 inhibition (Fig. 6B-D). This may be attributed to the complete inactivation of NF- $\mathrm{\kappa B}$ by p38 inhibition alone.

HFD feeding elevates hepatic cytokine production, which is reduced by myriocin or desipramine administration. To confirm whether A-SMase or CerS inhibition affects hepatic cytokine production in vivo, mice were fed an HFD for 24 weeks. Of note, 


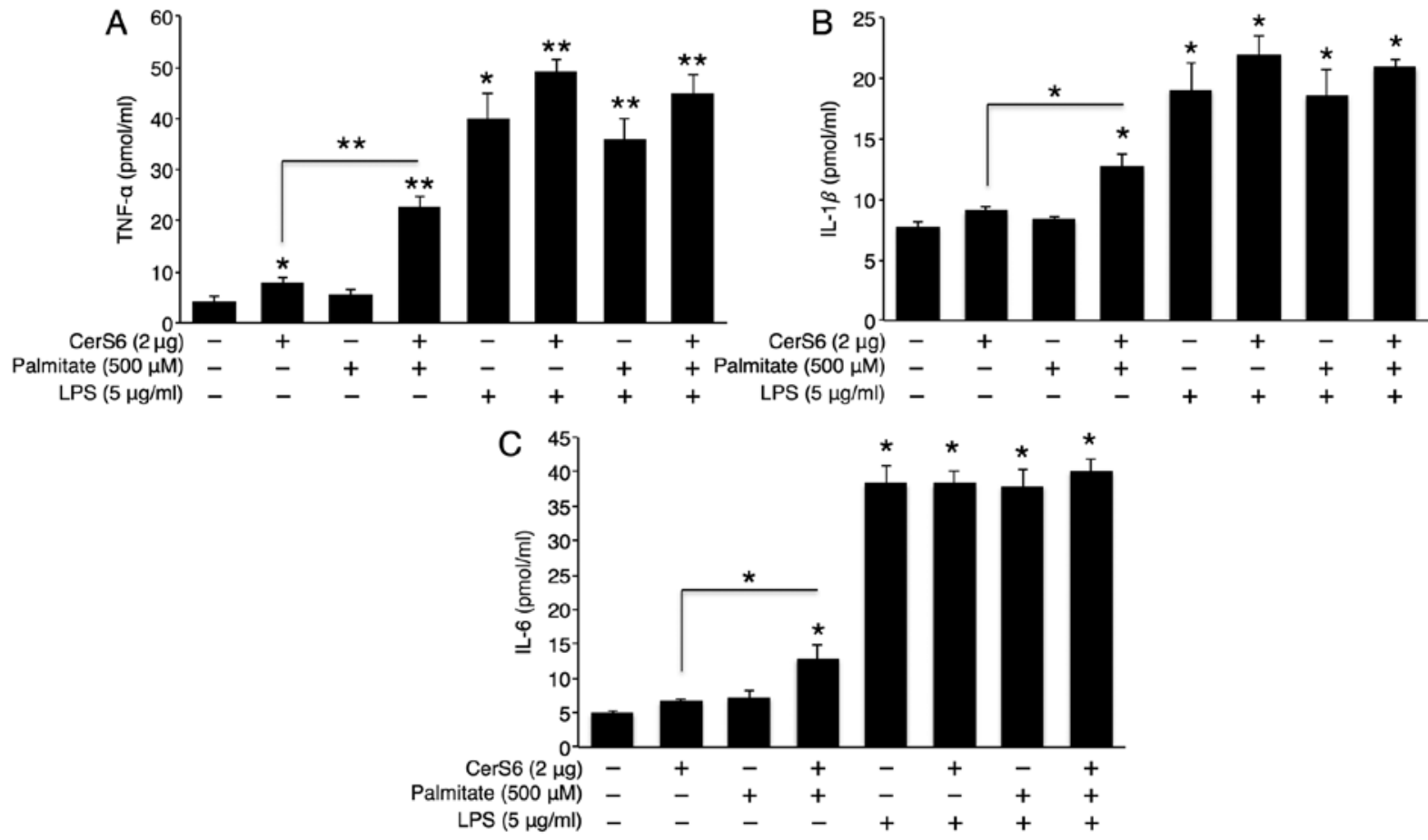

Figure 3. Neither palmitate treatment nor ceramide synthase 6 (CerS6) overexpression has synergistic effects with lipopolysaccharide (LPS) on the release of tumour necrosis factor (TNF)- $\alpha$, interleukin (IL)-1 $\beta$ and IL-6. Following co-treatment with palmitate and LPS, (A) TNF- $\alpha$, (B) IL-1 $\beta$, and (C) IL-6 levels were measured in culture medium from CerS6 overexpressing Hep3B cell using ELISA kits $(n=3)$. The values are expressed as the means \pm standard error of the mean. ${ }^{*} \mathrm{P}<0.05,{ }^{* * *} \mathrm{P}<0.01$. Three independent experiments were performed.

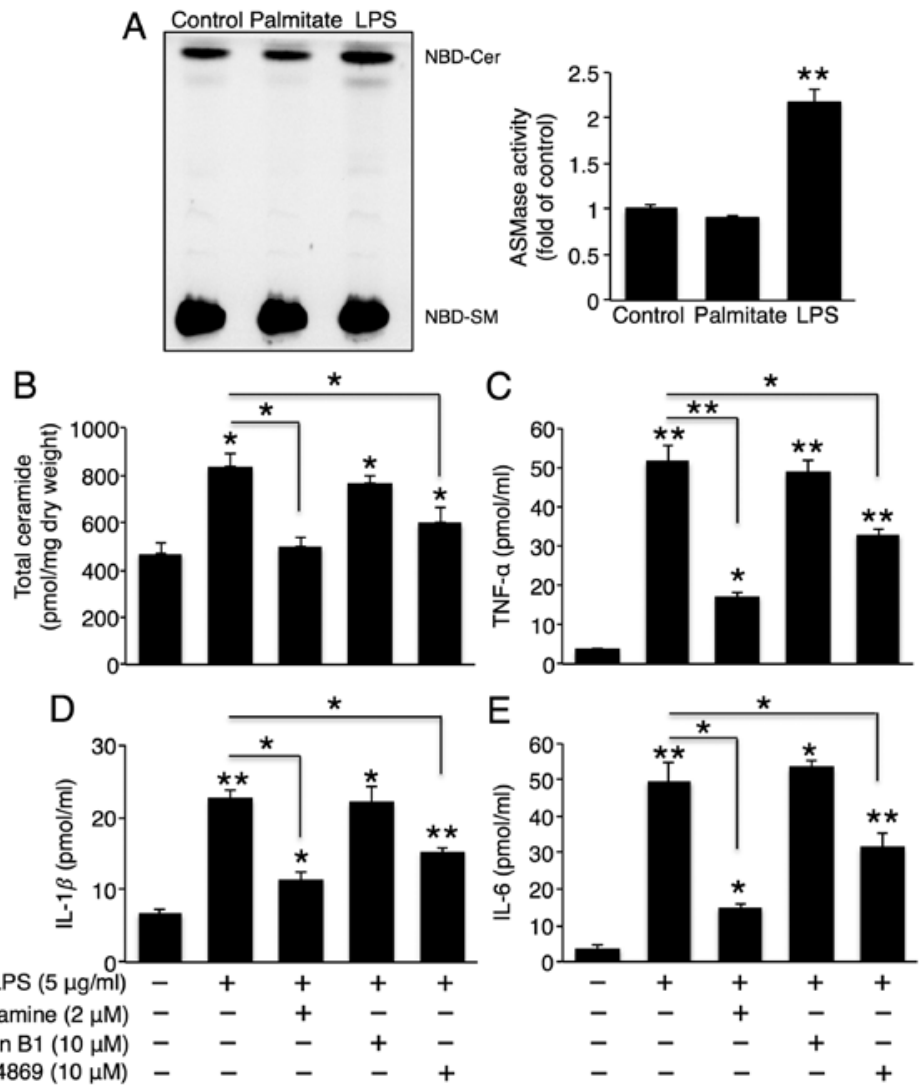

Figure 4. Sphingomyelin degradation plays an important role in the lipopolysaccharide (LPS)-induced secretion of tumour necrosis factor (TNF)- $\alpha$, interleukin (IL)-1 $\beta$ and IL-6. (A) Representative thin-layer chromatography of acidic sphingomyelinase (A-SMase) activity upon treatment with $500 \mu \mathrm{M}$ palmitate or $5 \mu \mathrm{g} / \mathrm{ml}$ LPS (left panel), as well as their quantification (right panel) (n=3). Following co-treatment with LPS, desipramine (A-SMase inhibitor), fumonisin B1 (CerS inhibitor), and GW4869 (neutral sphingomyelinase inhibitor), (B) total ceramide levels were measured using ESI-MS-MS (n=3), and (C) TNF- $\alpha$, (D) IL-1 $\beta$, and (E) IL-6 levels in Hep3B cell culture medium were measured using ELISA kits $(n=3)$. The values are expressed as the means \pm standard error of the mean. ${ }^{*} \mathrm{P}<0.05,{ }^{* *} \mathrm{P}<0.01$. Three independent experiments were performed. CerS, ceramide synthases. 
A

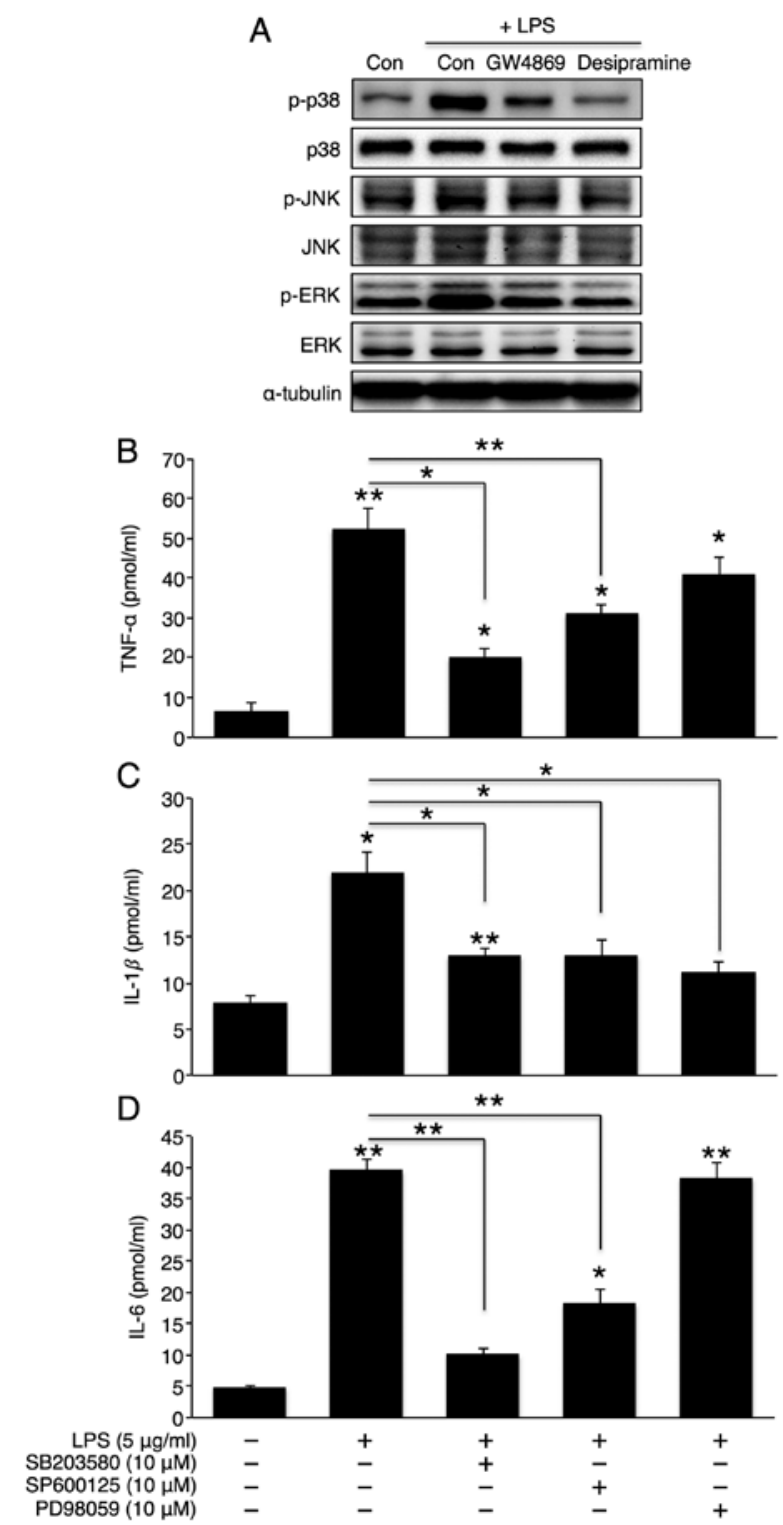

Figure 5. Mitogen-activated protein kinase (MAPK) activation plays a critical role in lipopolysaccharide (LPS)-induced cytokine secretion via sphingomyelin degradation. (A) Representative western blots of the MAPK pathway in LPS $(5 \mu \mathrm{g} / \mathrm{ml})$-treated Hep3B cells that were co-incubated with GW4869 (neutral sphingomyelinase inhibitor, $10 \mu \mathrm{M}$ ) or desipramine (an A-SMase inhibitor, $2 \mu \mathrm{M}$ ). Following co-treatment with LPS and SB203580 (p38 inhibitor), SP600125 (JNK inhibitor), or PD98059 (ERK inhibitor), (B) TNF- $\alpha$, (C) IL-1 $\beta$, and (D) IL-6 levels in Hep3B cell culture medium were measured using ELISA kits $(\mathrm{n}=3)$. The values are expressed as the means \pm standard error of the mean. ${ }^{*} \mathrm{P}<0.05,{ }^{* * *} \mathrm{P}<0.01$. Three independent experiments were performed. A-SMase, acidic sphingomyelinase.

the HFD increased CerS6 protein expression and decreased CerS2 protein expression in the liver (Fig. 7A), which caused a concomitant increase in C16-ceramide and a decrease in C24-ceramide (data not shown). Along with the HFD, desipramine or myriocin was intraperitoneally injected to inhibit A-SMase or de novo CerS, respectively. Due to the severe hepatotoxicity of FB1 treatment (30), an injection of myriocin, which is an inhibitor of serine-palmitoyl transferase, was used to inhibit de novo CerS. Desipramine or myriocin injection not only reduced p38, JNK and IкB phosphorylation (Fig. 7B), but also reduced hepatic TNF- $\alpha$, IL- $1 \beta$ and IL-6 production (Fig. 7C and D).
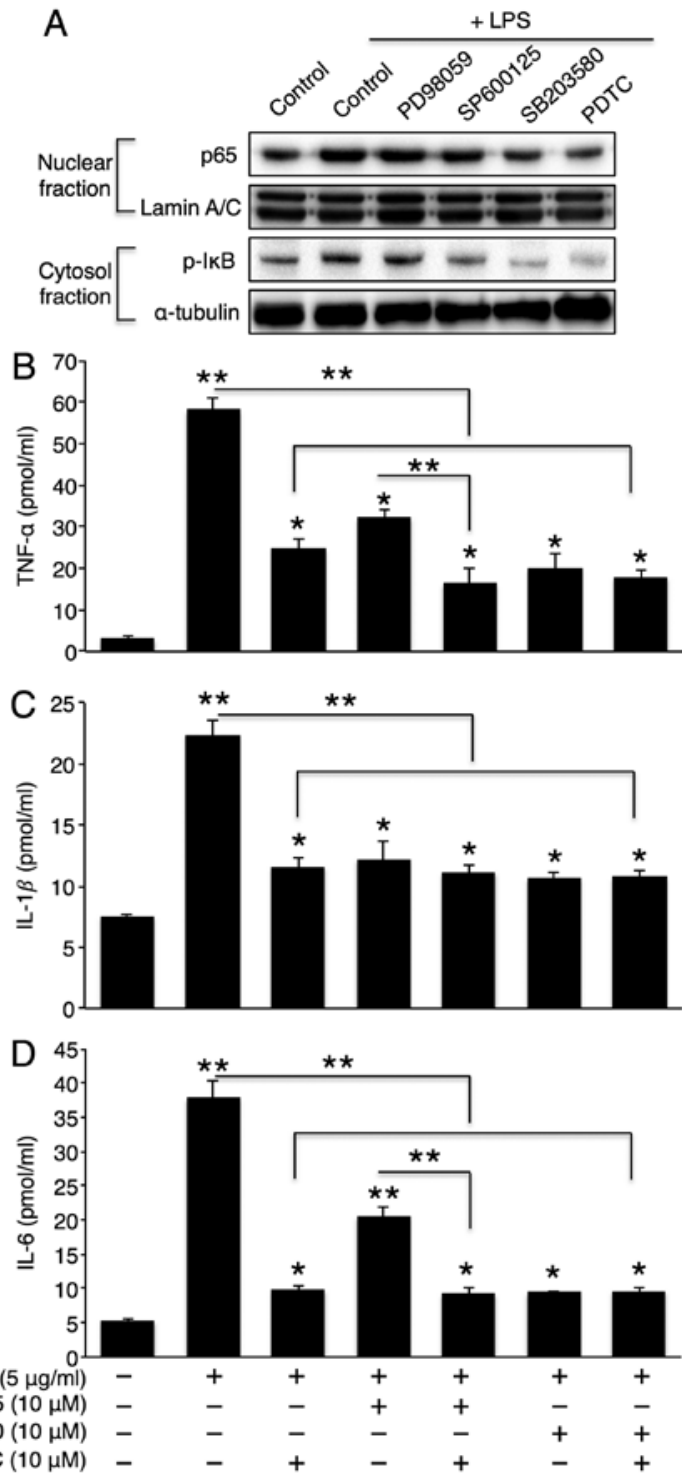

Figure 6. NF-kB activation plays a critical role in lipopolysaccharide (LPS)induced cytokine secretion. (A) Representative western blots of the NF- $\mathrm{kB}$ pathway in LPS $(5 \mu \mathrm{g} / \mathrm{ml})$-treated Hep3B cells that were co-incubated with $10 \mu \mathrm{M}$ PD98059 (ERK inhibitor), $10 \mu \mathrm{M}$ SP600125 (JNK inhibitor), $10 \mu \mathrm{M}$ SB203580 (p38 inhibitor), or $10 \mu \mathrm{M}$ PDTC (NF- $\kappa B$ inhibitor). Following co-treatment with LPS and SB203580, SP600125, or PDTC, (B) tumour necrosis factor (TNF)- $\alpha,(\mathrm{C})$ interleukin (IL)-1 $\beta$, and (D) IL-6 levels in Hep3B cell culture medium were measured using ELISA kits $(n=3)$. The values are expressed as the means \pm standard error of the mean. ${ }^{*} \mathrm{P}<0.05,{ }^{* * *} \mathrm{P}<0.01$. Three independent experiments were performed. PDTC, pyrrolidinedithiocarbamate ammonium.

\section{Discussion}

Obesity, which usually accompanies chronic inflammation (31), increases the risk of mortality and complications caused by diabetes. Various bioactive lipids, such as saturated fatty acids, cholesterol, and sphingolipids, have been proposed as mechanisms to explain the association between increased adiposity and the onset of these pathologies (32). Among these, ceramide has recently received attention as a key factor that is involved in the development of metabolic disorders, as the inhibition of ceramide formation ameliorates insulin resistance, atherosclerosis and cardiomyopathy $(32,33)$. In the present study, 
A

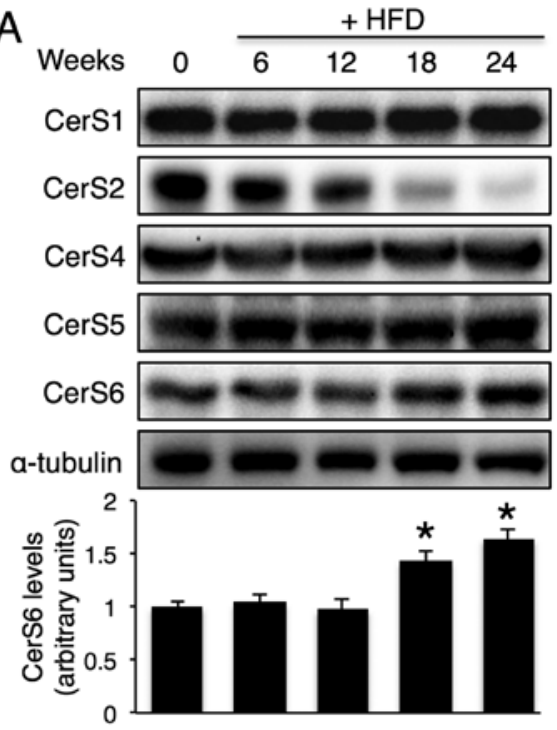

B

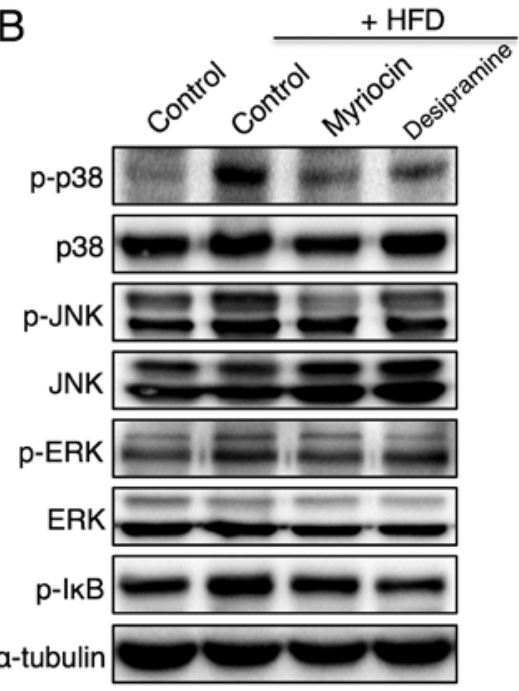

C

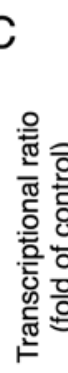

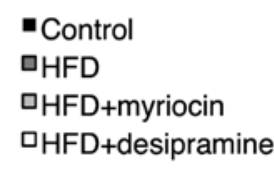

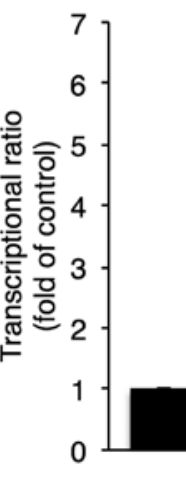
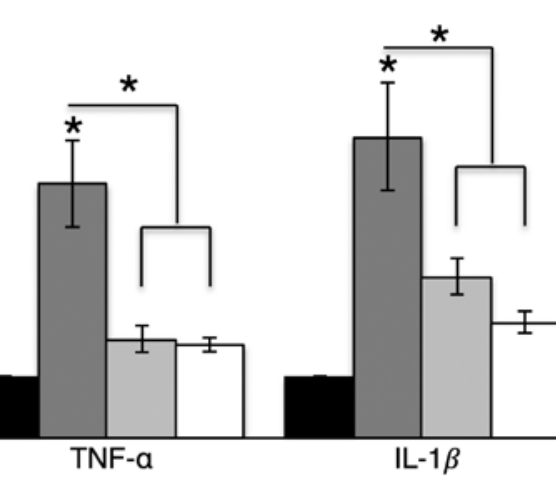

D

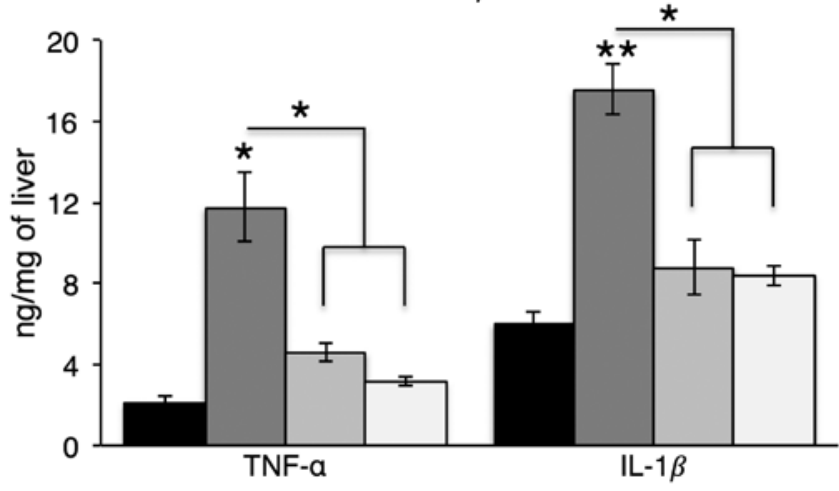

Figure 7. High-fat diet (HFD)-induced cytokine generation was inhibited by desipramine or myriocin injection. (A) Representative western blots of ceramide synthases (CerS) in the livers of mice after 6-24 weeks of being fed a HFD. (B) Representative western blots of mitogen-activated protein kinase (MAPK) pathways after 24 weeks HFD feeding with desipramine ( $10 \mathrm{mg} / \mathrm{kg} /$ day) or myriocin $(0.15 \mathrm{mg} / \mathrm{kg} /$ day), (C) relative expression of tumour necrosis factor (TNF)- $\alpha$, interleukin (IL)-1 $\beta$, and IL-6, (D) TNF- $\alpha$ and IL-1 $\beta$ levels in livers of HFD-fed mice that were co-treated with desipramine (10 mg/kg/day) or myriocin (0.15 mg/kg/day). The values are expressed as the means \pm standard error of the mean. ${ }^{*} \mathrm{P}<0.05,{ }^{* *} \mathrm{P}<0.01$. Three independent experiments were performed.

we further investigated whether ceramide formation plays a role in hepatic inflammation induced by palmitate, which is a saturated fatty acid, and LPS, which is a powerful bacterial virulence factor (34).

Six mammalian CerS produce ceramides that carry specific acyl chain lengths $(8,9)$. In this study, only CerS6 overexpression, which elevated C16-ceramide, increased TNF- $\alpha$ secretion from Hep3B cells. This confirmed a distinct role of ceramide that depended on the length of the acyl chain. Although the C2-ceramide has been reported to activate p38 MAPK and JNK in a T-cell line (35), in this study, CerS6 overexpression in Hep3B cells only activated p38 MAPK. Thus, a ceramide may activate different kinases depending on its acyl chain length. Of note, CerS5 overexpression, which also increased the C16-ceramide, did not affect TNF- $\alpha$ secretion. The difference between CerS5 and CerS6 has not yet been fully disclosed, although recent studies suggest that CerS5 and CerS6 play different roles $(36,37)$. For example, CerS5, but not CerS6, reduces fatty acid transport protein 5 expression. CerS6, but not CerS5, diminishes fatty acid binding protein 1 expression in Hep3B cells (36), and myristate-induced cardiomyocyte hypertrophy is dependent on CerS5, and not CerS6 (37). The exact mechanisms behind the different roles of CerS5 and CerS6 require further elucidation. 
In this study, TNF- $\alpha$ generation was regulated by the p38/NF- $\kappa$ B pathway, and both palmitate treatment in CerS6 overexpressing cells and LPS treatment elevated the phosphorylation of p38 and the nuclear translocation of p65. However, the initial steps differed. Treatment of the CerS6 overexpressing cells with palmitate activated p38 MAPK via de novo ceramide generation, whereas LPS stimulated p38 MAPK via A-SMase activation. Similar to the present study, an increase in C16-ceramide, which correlates with CerS6 elevation, has been shown to mediate IFN- $\gamma$-induced TNF- $\alpha$ release in RAW 264.7 macrophages (38). Thus, our data confirmed the general role of CerS6 in TNF- $\alpha$ generation.

LPS is found on the outer surface of Gram-negative bacteria. It can stimulate Toll-like receptors and generate various cytokines that stimulate the host response, and may lead to septic shock (28). In this study, the effect of LPS on the secretion of pro-inflammatory cytokines, including TNF- $\alpha$, IL- $1 \beta$ and IL-6 was robust. There was also no synergistic effect observed between palmitate and LPS as regards cytokine secretion by Hep3B cells. A previous study demonstrated synergistic effects between palmitate and LPS on IL-6 generation in macrophages (39). The synergistic effects of palmitate and LPS may differ depending on cell type, and these differences can be derived from distinct sphingolipid metabolism upon palmitate and LPS treatment. In macrophages, treatment with LPS and palmitate increases ceramide biosynthesis via the de novo pathway (40) and sphingomyelin degradation (39). However, in Hep3B cells, treatment with palmitate alone activated de novo ceramide generation. LPS only activated A-SMase, as FB1 treatment did not reduce LPS-induced cytokine release.

Similar to previous reports $(39,41)$, in this study, LPS activated A-SMase in Hep3B cells, and ceramide generation via A-SMase played a key role in cytokine secretion. Of note, the inhibition of N-SMase also partially reduced cytokine generation by inhibiting LPS-induced MAPK activation. This suggests that the activation of both A-SMase and N-SMase, but not CerS, plays a role in LPS-induced cytokine generation in the liver. Therefore, SMase inhibition may be a good therapeutic target in LPS-induced liver inflammation.

In the present study, we demonstrated that CerS6 generated C16-ceramide, which increased TNF- $\alpha$ secretion in liver cells. Furthermore, palmitate and CerS6 had synergistic effects on TNF- $\alpha$ secretion via de novo ceramide biosynthesis. Unlike palmitate stimulation, ceramide generation via sphingomyelin degradation played a key role in LPS-induced inflammatory cytokine production. Finally, the suppression of ceramide generation via A-SMase inhibition or de novo CerS decreased HFD-induced hepatic cytokine production in vivo. In conclusion, regulating hepatic ceramide generation may prove to be an effective therapeutic target for controlling hepatic inflammatory processes, and specific targets should differ depending on the inflammatory stimuli.

\section{Acknowledgements}

This study was supported by the Gachon University research fund of 2015 (no. GCU-2015-5112), Gachon University Gil Medical Center (no. GIL2012-01) and by grants from the Health Technology R\&D Project (no. HI14C2445) of the Ministry of Health and Welfare, Republic of Korea.

\section{References}

1. Esser N, Legrand-Poels S, Piette J, Scheen AJ and Paquot N: Inflammation as a link between obesity, metabolic syndrome and type 2 diabetes. Diabetes Res Clin Pract 105: 141-150, 2014.

2. Monteiro R and Azevedo I: Chronic inflammation in obesity and the metabolic syndrome. Mediators Inflamm 2010: 289645, 2010.

3. Boden G: Obesity, insulin resistance and free fatty acids. Curr Opin Endocrinol Diabetes Obes 18: 139-143, 2011.

4. Ståhlman M, Pham HT, Adiels M, Mitchell TW, Blanksby SJ, Fagerberg B, Ekroos K and Borén J: Clinical dyslipidaemia is associated with changes in the lipid composition and inflammatory properties of apolipoprotein-B-containing lipoproteins from women with type 2 diabetes. Diabetologia 55: 1156-1166, 2012.

5. Pillon NJ, Azizi PM, Li YE, Liu J, Wang C, Chan KL, Hopperton KE, Bazinet RP, Heit B, Bilan PJ, et al: Palmitate-induced inflammatory pathways in human adipose microvascular endothelial cells promote monocyte adhesion and impair insulin transcytosis. Am J Physiol Endocrinol Metab 309: E35-E44, 2015.

6. Haus JM, Kashyap SR, Kasumov T, Zhang R, Kelly KR Defronzo RA and Kirwan JP: Plasma ceramides are elevated in obese subjects with type 2 diabetes and correlate with the severity of insulin resistance. Diabetes 58: 337-343, 2009.

7. Brozinick JT, Hawkins E, Hoang Bui H, Kuo MS, Tan B, Kievit P and Grove K: Plasma sphingolipids are biomarkers of metabolic syndrome in non-human primates maintained on a Western-style diet. Int J Obes 37: 1064-1070, 2013.

8. Park WJ and Park JW: The effect of altered sphingolipid acyl chain length on various disease models. Biol Chem 396: 693-705, 2015.

9. Park JW, Park WJ and Futerman AH: Ceramide synthases as potential targets for therapeutic intervention in human diseases. Biochim Biophys Acta 1841: 671-681, 2014.

10. Venkataraman K, Riebeling C, Bodennec J, Riezman $\mathrm{H}$, Allegood JC, Sullards MC, Merrill AH Jr and Futerman AH: Upstream of growth and differentiation factor 1 (uog1), a mammalian homolog of the yeast longevity assurance gene 1 (LAG1), regulates N-stearoyl-sphinganine (C18-(dihydro) ceramide) synthesis in a fumonisin B1-independent manner in mammalian cells. J Biol Chem 277: 35642-35649, 2002.

11. Lahiri S and Futerman AH: LASS5 is a bona fide dihydroceramide synthase that selectively utilizes palmitoyl-CoA as acyl donor. J Biol Chem 280: 33735-33738, 2005.

12. Mizutani Y, Kihara A and Igarashi Y: Mammalian Lass6 and its related family members regulate synthesis of specific ceramides. Biochem J 390: 263-271, 2005.

13. Riebeling C, Allegood JC, Wang E, Merrill AH Jr and Futerman AH: Two mammalian longevity assurance gene (LAG1) family members, trh1 and trh4, regulate dihydroceramide synthesis using different fatty acyl-CoA donors. J Biol Chem 278: 43452-43459, 2003.

14. Laviad EL, Albee L, Pankova-Kholmyansky I, Epstein S, Park H, Merrill AH Jr and Futerman AH: Characterization of ceramide synthase 2: Tissue distribution, substrate specificity, and inhibition by sphingosine 1-phosphate. J Biol Chem 283: 5677-5684, 2008.

15. Mizutani Y, Kihara A and Igarashi Y: LASS3 (longevity assurance homologue 3 ) is a mainly testis-specific (dihydro) ceramide synthase with relatively broad substrate specificity. Biochem J 398: 531-538, 2006.

16. Mesicek J,Lee H,Feldman T, Jiang X, Skobeleva A, BerdyshevEV, Haimovitz-Friedman A, Fuks Z and Kolesnick R: Ceramide synthases 2,5 , and 6 confer distinct roles in radiation-induced apoptosis in HeLa cells. Cell Signal 22: 1300-1307, 2010.

17. Goñi FM and Alonso A: Sphingomyelinases: Enzymology and membrane activity. FEBS Lett 531: 38-46, 2002.

18. Tolman KG, Fonseca V, Dalpiaz A and Tan MH: Spectrum of liver disease in type 2 diabetes and management of patients with diabetes and liver disease. Diabetes Care 30: 734-743, 2007.

19. Roux JC, Dura E, Moncla A, Mancini J and Villard L: Treatment with desipramine improves breathing and survival in a mouse model for Rett syndrome. Eur J Neurosci 25: 1915-1922, 2007.

20. Glaros EN, Kim WS, Wu BJ, Suarna C, Quinn CM, Rye KA, Stocker R, Jessup W and Garner B: Inhibition of atherosclerosis by the serine palmitoyl transferase inhibitor myriocin is associated with reduced plasma glycosphingolipid concentration. Biochem Pharmacol 73: 1340-1346, 2007. 
21. Ali M, Fritsch J, Zigdon H, Pewzner-Jung Y, Schütze S and Futerman AH: Altering the sphingolipid acyl chain composition prevents LPS-GLN-mediated hepatic failure in mice by disrupting TNFR1 internalization. Cell Death Dis 4: e929, 2013.

22. Halasiddappa LM, Koefeler H, Futerman AH and Hermetter A: Oxidized phospholipids induce ceramide accumulation in RAW 264.7 macrophages: Role of ceramide synthases. PLoS One 8: e70002, 2013

23. Jeong YM, Park WJ, Kim MK, Baek KJ, Kwon NS, Yun HY and Kim DS: Leucine-rich glioma inactivated 3 promotes $\mathrm{HaCaT}$ keratinocyte migration. Wound Repair Regen 21: 634-640, 2013.

24. Livak KJ and Schmittgen TD: Analysis of relative gene expression data using real-time quantitative PCR and the 2(-Delta Delta C(T)) Method. Methods 25: 402-408, 2001.

25. Choi S, Kim JA, Kim TH, Li HY, Shin KO, Lee YM, Oh S, Pewzner-Jung Y, Futerman AH and Suh SH: Altering sphingolipid composition with aging induces contractile dysfunction of gastric smooth muscle via $\mathrm{K}(\mathrm{Ca}) 1.1$ upregulation. Aging Cell 14: 982-994, 2015.

26. Shaner RL, Allegood JC, Park H, Wang E, Kelly S, Haynes CA, Sullards MC and Merrill AH Jr: Quantitative analysis of sphingolipids for lipidomics using triple quadrupole and quadrupole linear ion trap mass spectrometers. J Lipid Res 50: 1692-1707, 2009.

27. Campbell J, Ciesielski CJ, Hunt AE, Horwood NJ, Beech JT Hayes LA, Denys A, Feldmann M, Brennan FM and Foxwell BM: A novel mechanism for TNF-alpha regulation by p38 MAPK: Involvement of NF-kappa B with implications for therapy in rheumatoid arthritis. J Immunol 173: 6928-6937, 2004

28. Józefowski S, Czerkies M, Łukasik A, Bielawska A, Bielawski J, Kwiatkowska K and Sobota A: Ceramide and ceramide 1-phosphate are negative regulators of TNF- $\alpha$ production induced by lipopolysaccharide. J Immunol 185: 6960-6973, 2010.

29. El Alwani M, Wu BX, Obeid LM and Hannun YA: Bioactive sphingolipids in the modulation of the inflammatory response. Pharmacol Ther 112: 171-183,2006.

30. Soriano JM, González L and Catalá AI: Mechanism of action of sphingolipids and their metabolites in the toxicity of fumonisin B1. Prog Lipid Res 44: 345-356, 2005.

31. Dandona P, Aljada A and Bandyopadhyay A: Inflammation: The link between insulin resistance, obesity and diabetes. Trends Immunol 25: 4-7, 2004

32. Bikman BT and Summers SA: Ceramides as modulators of cellular and whole-body metabolism. J Clin Invest 121: 4222-4230, 2011.
33. Holland WL, Brozinick JT, Wang L-P, Hawkins ED, Sargent KM, Liu Y, Narra K, Hoehn KL, Knotts TA, Siesky A, et al: Inhibition of ceramide synthesis ameliorates glucocorticoid-, saturated-fat-, and obesity-induced insulin resistance. Cell Metab 5: 167-179, 2007.

34. Blais DR, Vascotto SG, Griffith M and Altosaar I: LBP and CD14 secreted in tears by the lacrimal glands modulate the LPS response of corneal epithelial cells. Invest Ophthalmol Vis Sci 46: 4235-4244, 2005

35. Chen CL, Lin CF, Chang WT, Huang WC, Teng CF and Lin YS: Ceramide induces p38 MAPK and JNK activation through a mechanism involving a thioredoxin-interacting protein-mediated pathway. Blood 111: 4365-4374, 2008

36. Park WJ, Park JW, Merrill AH Jr, Storch J, Pewzner-Jung Y and Futerman AH: Hepatic fatty acid uptake is regulated by the sphingolipid acyl chain length. Biochim Biophys Acta 1841: 1754-1766, 2014

37. Russo SB, Baicu CF, Van Laer A, Geng T, Kasiganesan H, Zile MR and Cowart LA: Ceramide synthase 5 mediates lipid-induced autophagy and hypertrophy in cardiomyocytes. J Clin Invest 122: 3919-3930, 2012.

38. Schiffmann S, Ferreirós N, Birod K, Eberle M, Schreiber Y, Pfeilschifter W, Ziemann U, Pierre S, Scholich K, Grösch S and Geisslinger G: Ceramide synthase 6 plays a critical role in the development of experimental autoimmune encephalomyelitis. J Immunol 188: 5723-5733, 2012.

39. Jin J, Zhang X, Lu Z, Perry DM, Li Y, Russo SB, Cowart LA, Hannun YA and Huang Y: Acid sphingomyelinase plays a key role in palmitic acid-amplified inflammatory signaling triggered by lipopolysaccharide at low concentrations in macrophages. Am J Physiol Endocrinol Metab 305: E853-E867, 2013.

40. Schilling JD, Machkovech HM, He L, Sidhu R, Fujiwara H, Weber K, Ory DS and Schaffer JE: Palmitate and lipopolysaccharide trigger synergistic ceramide production in primary macrophages. J Biol Chem 288: 2923-2932, 2013.

41. Cuschieri J, Bulger E, Billgrin J, Garcia I and Maier RV: Acid sphingomyelinase is required for lipid Raft TLR4 complex formation. Surg Infect (Larchmt) 8: 91-106, 2007.

42. Liu G, Friggeri A, Yang Y, Park YJ, Tsuruta Y and Abraham E: miR-147, a microRNA that is induced upon Toll-like receptor stimulation, regulates murine macrophage inflammatory responses. Proc Natl Acad Sci USA 106: 15819-15824, 2009.

43. Kawane K, Tanaka H, Kitahara Y, Shimaoka S and Nagata S: Cytokine-dependent but acquired immunity-independent arthritis caused by DNA escaped from degradation. Proc Natl Acad Sci USA 107: 19432-19437, 2010. 